\title{
Electric Fields due to Meteoric Charged Dust in the Mesosphere
}

\author{
Victoria V. Yaroshenko ${ }^{1}$ and Marcel Goossens \\ Center for Plasma Astrophysics, K.U. Leuven, Belgium \\ Alexander P. Nickolaenko, \\ Usikov Institute for Radio-Physics and Electronics \\ Ukrainian National Academy of Sciences, Kharkov, Ukraine
}

\begin{abstract}
Gravitational sedimentation of charged dust and its subsequent discharging by ionospheric electrons and/or ions results in a peculiar plasma capacitor and a vertical polarization of electric fields within the upper mesosphere. This mechanism predicts the electric fields from several tens to a few hundreds of $\mathrm{mV} / \mathrm{m}$ at altitudes $85-100 \mathrm{~km}$.
\end{abstract}

Key words: dusty plasma, mesospheric electric field, meteor dust

\section{Introduction}

Most of the early work on dust in the Earth's environment concentrated on the role of the dust for the opacity of the atmosphere. Observations clearly pointed to the presence of numerous aerosols or dust particles at mesospheric altitudes, [Lebedinets, 1981]. Research in past decades has shown that most of the meteoric stall and ablation occurs at the height of about $100 \mathrm{~km}$. It is widely assumed that the vaporized material recondenses and forms aerosol particles, which then coagulate and slowly drift downward through the atmosphere. Following the theory, a dust particle immersed in plasma acquires a charge supplied by the flux of ions and electrons it absorbs. In a steady state charging model [Goertz, 1989], dust immersed in plasma consisting of only electrons and ions, where photoelectrons, secondary emission and other charging processes can be neglected, will develop a negative charge. Positive charges do relay upon photoelectric emission as the dominant mechanism. However, there are small number confirmed measurements of charged dust at the mesospheric altitudes.

\footnotetext{
${ }^{1}$ On leave from Institute of Radio Astronomy National Academy of Science of Ukraine
} 
The most promising reports so far are the detection of charged particles in noctilucent clouds (NLC) and in polar mesosphere summer echoes (PMSE) [Havnes et al., 1996, Havnes et al., 2001] and measurements in the tropical mesosphere, [Gelinas et al., 1998, Horanyi et al., 2000] by rocket-borne dust detectors. In particular, the rocket measurements of the polar mesosphere show that in NLC/PMSE conditions large amounts of charged dust must be present, with average sizes less than $0.1 \mathrm{\mu m}$, at densities of several $10^{2} \mathrm{~cm}^{-3}$. Both negatively and positively charged dust has been detected on the same rocket flight at different altitudes (82$85 \mathrm{~km}$ ). The rocket observations of the mesospheric charged dust in the tropical ionosphere indicate a $5 \mathrm{~km}$ thick layer of small charged dust (500-1000 amu), most likely of meteoric origin, at altitudes of $90-95 \mathrm{~km}$, in the vicinity of a sporadic Elayer $(92,5 \mathrm{~km})$. The observed dust was positively charged in the bulk of the dust layer, but was negatively charged near the bottom. A similar experiment (White Sands, New Mexico) found a $500 \mathrm{~m}$ positive layer at an altitude $86.5 \mathrm{~km}$ and a wide layer of negative charges below it [Horanyi et al., 2000]

Electromagnetic processes in ionospheric plasmas are traditionally associated with the electron and ion components. Since the late 1980 s there is an increasing interest in the role of dust in the mesospheric plasma (e.g. [Goldberg, 1989, Mitchel, 1990, Goldberg and Holzworth, 1991, Zadorozhny et al., 1994, Zadorozhny, 2000]). This trend is not surprising, as charged dust particles introduce new features in the plasma processes. Indeed, we are dealing with a massive component much heavier than the mass of the plasma electrons and ions. The latter can have multiple charges and introduce strong inhomogeneities on scales of the Debye length. Dust can act as a source or a sink for the electrons or ions, possibly introducing compositional changes. Moreover, dust particles are subject to non-electromagnetic forces, e.g., gravity, friction, etc. So we can expect meteoric dust to be especially important in the mesosphere, and first of all in view of its role in the generation of mesospheric vertical electric fields [Bragin et al., 1974, Tyutin, 1976, Zadorozhny et al., 1993, Zadorozhny et al., 1994, Hale et al., 1981, Maynard et al., 1981, Croskey et al., 1885]).

In particular, Aikin and Maynard [1990] analyzed the electric fields due to the gravitational falling of charged meteoric particles through the low ionosphere. The vertical field was obtained from a local current balance between the downward flux of charged dust and conduction current. This model, however, predicts maximum fields of the order $10 \mathrm{mV} / \mathrm{m}$ at the low altitude of about $65 \mathrm{~km}$ because electric field dramatically decreases above $70 \mathrm{~km}$ altitude due to high conductivity of the ionosphere. The dust was also considered as a composition of multiply positively and negatively charged particles of different sizes [Zadorozhny, 2000]. The heavier species falling with larger velocities initiate a charge separation, while the smaller ones reduce the effective conductivity of the plasma, thus providing a large vertical 
electric field reaching the $\mathrm{V} / \mathrm{m}$ level. However, this model is not truly realistic. The main assumption that numerous positively and negatively highly charged dust particles with charge densities much larger than those of electrons and ions at the same mesospheric altitudes exist together is not proven experimentally. On the contrary, the observational data demonstrate layers of likely charged dust [Havnes et a.,l 1996, Havnes et al., 2001, Gelinas et al., 1998, Horanyi et al., 2000].

In this paper we present a simple model for the generation of electric fields in the ionospheric plasma. We build our treatment on the existence of a dust layer at upper mesospheric altitudes, and the dust particles are electrically charged mainly by the same charging mechanism. We show that a charge separation in the Earth's gravitational field gives rise to a peculiar plasma capacitor and to a plasma polarization in the electric field. The latter is strongly dependent on the dust size and its density: By using reasonable parameters for the dust component, we evaluate the electric field strength that is found between several tens and a few hundreds of $m V / m$, which is comparable with the local plasma threshold field $E_{p}=\sqrt{\frac{3 \kappa T}{e^{2}} m_{e} \delta v^{2}} \approx 10^{-4} \mathrm{~V} / \mathrm{m}$. Here, $\kappa$ is the Boltzmann constant, $m_{\mathrm{e}}$ and $e$ are the electron mass and charge, $\delta \leq 10^{-3}$ is the fraction of energy lost by electron in a collision with heavy particle, $v$ is the effective collision frequency of electrons.

\section{A Capacitor Model for the Electric Field Generation}

We assume that there is a dust layer of a $\mathrm{d} \approx 1 \mathrm{~km}$ thickness located at the mesospheric altitudes between 80 and $100 \mathrm{~km}$. Of course, a very low density dust component is present throughout the bulk of the ionosphere and atmosphere but meteor ablation supplies the fine dust particles to these altitudes. The dusty plasma layer is neutral:

$$
e N_{e}+q_{i} N_{i}+q_{d} N_{d}=0
$$

where $e, q_{\mathrm{i}}$, and $q_{\mathrm{d}}$ are the particle charges; $N_{\mathrm{e}}, N_{\mathrm{i}}$, and $N_{\mathrm{d}}$ are the undisturbed electron, ion, and dust densities in the layer. The dusty stratum is characterized by a mono-sized grain population for simplicity. We will specify the sign and values $q_{\mathrm{d}}$ later, when considering particular regions of the mesosphe. The electron and ion plasma densities outside the dust layer are assumed to be equal: $N_{e}=N_{i}=N_{0}$

Since the grains are relatively massive, they remain essentially nonmagnetized and slowly advance downward through the atmosphere driven by gravity force. In contrast, the magnetized electrons and ions are able to move only along the magnetic field lines. As a result, the ensuing charge separation and the arising electric field will be strongly dependent on the direction of the geomagnetic field. 
Having formulated these conditions, we can consider special cases corresponding to the horizontal and oblique geomagnetic field $H_{0}$ and to different signs of the dust charge.

\subsection{Case1: Tropical Mesosphere}

First of all, we consider a tropical mesosphere by assuming that $H_{0}$ is horizontal and parallel to the dust layer. The charged dust component drifts to the Earth under the action of gravity and carries away the electric charge, while electrons and ions are not free to follow them. Thus the plasma quasi-neutrality condition (1) is violated. The charged dust particle cannot penetrate far away into the underlying ionospheric plasma because of its collisions with electrons and ions. These latter have the number densities much higher than that of the dusty particles. The grain becomes a neutral particle by swallowing up electrons (ions) thus producing a charge imbalance $\left(N_{\mathrm{i}} \neq N_{\mathrm{e}}\right)$ below the initial dust layer. The distance $D$ that the charged dust particle can travel depends on its velocity $V_{\mathrm{d}}$ and the recharging (neutralization) time $t_{\mathrm{C}}$. The dust velocity $V_{\mathrm{d}}$ for a spherical particle of radius $a$ is given by [Jensen and Thomas, 1988]:

$$
V_{d}=\frac{g a}{2 p} \rho_{d} \sqrt{\frac{\pi \kappa T}{2 m_{E}}}
$$

Here, $p$ is the ambient pressure, $\rho_{\mathrm{d}}$ is the mass density of the grain, $g$ the acceleration of gravity; and $m_{\mathrm{E}}$ refers to the mass of a typical atmospheric molecule. The timescale $t_{\mathrm{C}}$ for a dust grain neutralization by electrons (ions) is obtained from [Goertz, 1989]:

$$
t_{C}=\frac{1}{4 \pi a^{2} N_{\alpha} V_{T_{\alpha}}}
$$

where $N_{\alpha}$ is the density and $V_{T_{\alpha}}$ is the thermal velocity of either electrons ( $\alpha=e$, when the sign of the dust charge is positive) or ions ( $\alpha=i$ in the case of negatively charged dust).

Then, the grain path $D$ can be roughly estimated as $D \approx V_{d} t_{C}$. In this picture, the spatial charge separation arises due to dust sedimentation caused by the gravitational field. It produces a "plasma capacitor" with two parallel oppositely charged layers ("plates") separated by some distance, which is of the order of either the thickness of the initial dusty layer $d$ (in the case when $d>>D$ ) or of $D$ (when $d$ $<<D$ ). The "upper plate" carries an excess of ionospheric particles having the 
opposite sign compared to the dust charges, whereas the potential of the "lower plate" is conditioned by the charged grains. As a result, the vertical electric field is polarized in the direction of the gravitation force for the negatively charged dust or in the opposite direction for the positive charged dust.

To apply the analysis to real plasmas we use the observational data for the tropical region [Gelinas et al., 1998]; which imply that the probability for acquiring the minimum excess charge by a dust particle is not very high. The meteoric dust model predicts dust sizes of $a \sim 1-10 \mathrm{~nm}$ at a density of $10^{3} \mathrm{~cm}^{-3}$ at the altitudes of about $90 \mathrm{~km}$. In order not to overestimate the role of the charged dust, we assume that only a few percents of the dust particles carry the minimum excess positive charge, that is $N_{\mathrm{d}} \sim 10 \mathrm{~cm}^{-3}$ and $q_{\mathrm{d}}=q_{\mathrm{dmin}}=-e>0$. This case is relevant to the daylight hours, when the particles become positively charged by the photoelectric emission. The electron density and thermal velocity are about $10^{4} \mathrm{~cm}^{-3}$ and $V_{T_{e}}=$ $4 \times 10^{4} \mathrm{~m} / \mathrm{s}$ respectively and the characteristic time $t_{C}(3)$ ranges from a few seconds to a few minutes, depending on the particle size (see Table 1). This implies the distance $D$ ranging from 2.5 to $0.25 \mathrm{~m}$, being, on the one hand, much shorter than the initial layer thickness $d$ and on the other, much greater than the Debye length pertinent to the mesospheric plasma that reaches usually a few centimeters. The electric field between the "plasma capacitor plates" can be estimated as:

$$
E=\frac{\sigma}{\varepsilon_{0}} \cong \frac{e N_{d} D}{\varepsilon_{0}}
$$

where the value $\sigma=e N_{\mathrm{d}} D$ represents the effective surface density of the charges.

Table 1. Tropical mesosphere, the $90 \pm 2 \mathrm{~km}$ altitudes, $q_{\mathrm{d}}=-e$

\begin{tabular}{ccccc}
\hline$\alpha, n m$ & $t_{\mathrm{C},} s$ & $V_{\mathrm{d}}, \mathrm{cm} / s$ & $D, m$ & $E, V / m$ \\
\hline 1 & 199 & 1.3 & 2.56 & 0.46 \\
2 & 49.8 & 2.6 & 1.29 & 0.23 \\
4 & 12.4 & 5.2 & 0.645 & 0.12 \\
6 & 5.53 & 7.8 & 0.431 & 0.08 \\
8 & 3.11 & 10.4 & 0.323 & 0.058 \\
10 & 1.99 & 13 & 0.258 & 0.046 \\
\hline
\end{tabular}

Table 1 lists the vertical electric fields corresponding to the grains of different size in ambient daytime conditions at the altitudes of $90-95 \mathrm{~km}$. It turns out, that the 
dust grains with a size of a few $n m$, can easily generate local electric fields of several tens or even of hundreds of $\mathrm{mV} / \mathrm{m}$, as was frequently reported in experiments.

The situation can be totally different during the nighttime. Now, the dust is expected to be negatively charged: $q_{\mathrm{d}}=q_{\mathrm{d} \min }=e<0$. The neutralization time (3) is increased considerably reaching $t_{\mathrm{C}} \sim 10^{2}-10^{4} \mathrm{~s}$ at altitudes of about $90 \mathrm{~km}$, conditioned by $V_{T i} \gg V_{T e}$. As a result, $D$ increases and the resulting imbalance in charge densities causes electric fields, much stronger than in the daytime. The field is directed downward, to the Earth. We show the parameters and the electric field obtained in Table 2 .

Table 2. Tropical mesosphere, the $90 \pm 2 \mathrm{~km}$ altitudes, $q_{\mathrm{d}}=e$

\begin{tabular}{ccccc}
\hline$\alpha, n m$ & $t_{\mathrm{C}, s}$ & $V_{\mathrm{d}}, \mathrm{cm} / \mathrm{s}$ & $D, m$ & $E, V / m$ \\
\hline 1 & $7.96 \times 10^{3}$ & 1.3 & 103.48 & 18.62 \\
2 & $199 \times 10^{3}$ & 2.6 & 51.74 & 9.31 \\
4 & $49.7 \times 10^{2}$ & 5.2 & 25.84 & 4.64 \\
6 & $12.4 \times 10^{2}$ & 7.8 & 17.23 & 3.1 \\
8 & $5.53 \times 10^{2}$ & 10.4 & 12.9 & 2.3 \\
10 & 79.6 & 13 & 10.35 & 1.86 \\
\hline
\end{tabular}

\subsection{Case 2: Polar Mesosphere}

In the case of an oblique magnetic field superimposed on the dusty layer, the plasma electrons and ions may follow the charged grains by moving along the field lines until the split between their paths will exceed the Debye length $\lambda_{D}$. It is easy to see that the charge separation corresponds to the distance $\Delta D=\lambda_{D} / \cos \chi$ that depends on the tilt of the geomagnetic field $\chi$. Hence, the "plasma capacitor" may exist only inside the dusty layer. In contrast to the tropic case, the polar capacitor carries a smaller effective charge density $\sigma=e N_{d}[D-\Delta D]$, and relevant electric field (4) is reduced. Obviously, the vertical electric field cannot be generated in the immediate vicinity of the magnetic pole where $\chi \rightarrow \frac{\pi}{2}$ giving $\Delta D \rightarrow \infty, \sigma \rightarrow 0$, and $E \rightarrow 0$.

We estimate the vertical electric field, generated in the polar mesosphere at the altitudes of $82-85 \mathrm{~km}$ by assuming that an angle of inclination is $\chi \sim 80^{\circ}$ and the ionosphere is characterized by $N_{0}=5 \times 10^{3} \mathrm{~cm}^{-3}, V_{T e}=2.15 \times 10^{4} \mathrm{~m} / \mathrm{s}$, and $V_{T i}=$ $5 \times 10^{2} \mathrm{~m} / \mathrm{s}$. According to measurements in the polar mesosphere summer echoes 
(PMSE) conditions, the dust charge density is sufficiently high at these altitudes: $N_{d} Z_{d}=N_{d} q_{d} / e \sim 2 \times 10^{2} \mathrm{~cm}^{-3}$. [Havnes et al. 1996, Zadorozhny, 2000], and the dust sizes are of the order $a \sim 10-50 \mathrm{~nm}$ [Gumbel et al., 2001]. The computed electric fields (4) are listed in Table 3 and Table 4 for respectively $q_{\mathrm{d}}=-e>0$ and $q_{\mathrm{d}}=e<0$.

Table 3. Polar mesosphere, the $82-85 \mathrm{~km}$ altitudes, $q_{\mathrm{d}}=-e$

\begin{tabular}{ccccc}
\hline$\alpha, n m$ & $t_{\mathrm{C},} s$ & $V_{\mathrm{d}}, m / s$ & $D-\Delta D, m$ & $E, V / m$ \\
\hline 8 & 12.44 & 0.1 & 1.12 & 0.43 \\
10 & 7.96 & 0.13 & 0.93 & 0.33 \\
20 & 1.99 & 0.26 & 0.31 & 0.13 \\
30 & 0.88 & 0.39 & 0.25 & 0.08 \\
40 & 0.5 & 0.52 & 0.15 & 0.06 \\
50 & 0.32 & 0.65 & 0.1 & 0.03 \\
\hline
\end{tabular}

Table 4. Polar mesosphere, the $82-85 \mathrm{~km}$ altitudes, $q_{\mathrm{d}}=e$

\begin{tabular}{ccccc}
\hline$\alpha, n m$ & $t_{\mathrm{C},} s$ & $V_{\mathrm{d}}, m / s$ & $D-\Delta D, m$ & $E, V / m$ \\
\hline 10 & 636.8 & 0.13 & 82.69 & 14.87 \\
15 & 282.67 & 0.195 & 55.01 & 9.91 \\
20 & 159.2 & 0.26 & 41.29 & 7.43 \\
30 & 70.76 & 0.39 & 27.48 & 4.94 \\
40 & 39.8 & 0.52 & 20.59 & 3.69 \\
50 & 25.47 & 0.65 & 16.45 & 2.92 \\
\hline
\end{tabular}

\section{Conclusion}

The mesospheric plasma contains layers of charged grains whose drift may generate the electric fields in the mesosphere. By using reasonable parameters for the dust component, we have obtained values for the electric field strength between several tens and a few hundreds of $\mathrm{mV} / \mathrm{m}$.

We have used a simplified model with a mono-sized dust component. Still, one may expect that the introduction of a particle size distribution will qualitatively modify our results. When there are a few dusty layers in the atmosphere, each of them will form a capacitor at relevant altitude, so that a complex distribution of the vertical electric fields will be generated. 
The simple approach outlined in this paper indicates on several effects.

1). A vertical electric field resulting from sedimentation of the charged dust can substantially exceed the value of the plasma threshold field in the lower ionosphere $\sim 10^{-4} \mathrm{~V} / \mathrm{m}$. This means that presence of charged dust can play an important role in the initiation and support of non-linear processes in the upper mesosphere.

2). The direction and magnitude of the vertical electric field may vary with the time of day. Quite possibly, this may cause mesospheric electric currents flowing at relevant latitudes. Such electrification and the currents may be different at low and high latitudes.

Authors hope that the results presented will stimulate further experimental studies of the electric fields in the mesosphere. Detection of the static electric fields or related nonlinear effects pertinent to the $85-100 \mathrm{~km}$ altitudes will provide an evidence of the role of the charged dust plays in terrestrial electrodynamics.

\section{Acknowledgments}

VY thanks the "Onderzoeksfonds K.U. Leuven" for supporting her through a research fellowship grant No. F/00/070.

\section{REFERENCES}

Aikin, A.C., N.C. Maynard, and A Van de Graaf, Source méechanism for middle atmospheric vertical electric fields, J. Atmos. Terr. Phys., 52, 695-705, 1990.

Bragin, Yu. A., A.A. Tyutin, and A.A. Kocheev, Direct measurements of the atmospheric vertical electric field strength up to $80 \mathrm{~km}$, Cosmic Res., Eng. Trans., 12, 279-280, 1974.

Croskey, C.L. , L.C. Hale, J.D. Mitchel, D. Muha, and N.C. Maynard, A diurnal study of the electrical structure of the equatorial middle atmosphere, J. Atmos. Terr. Phys., 47, 835-844, 1985.

Gelinas, L.J., K.A. Lynch, M.C. Kelley, S. Collins, S. Baker, Q. Zhou, and J.S. Friedman, First observation of meteoric charged dust in the tropical mesosphere, Geophys. Res. Lett., 25, 4047-4050, 1998.

Goertz, C.K., Dusty plasmas in the Solar system, Rev. Geophys., 27, 271-292, 1989.

Goldberg R.A., Electrodynamics of the high latitude mesosphere, J. Geophys. Res., 94, 14,661-14,672, 1989.

Goldberg R.A. and R.H. Holzworth, Middle atmospheric electrodynamics, Handbook for MAP, 32, 63-84, 1991. 
Gumbel J., J. Stegman, D.P.Murtagh, and G. Witt, Scattering phase function and particle sizes in noctilucent clouds, Geophys. Res. Lett., 28, 1415-1418, 2001.

Hale L.C., Croskey, C.L., and J.D. Mitchel, Measurements of middle atmosphere electric field and associated electrical conductivities, Geophys. Res. Lett., 8, 927-931, 1981.

Havnes, O., J. Troeim, T. Blix, W. Mortensen, L.I. Naesheim, E. Thrane, and Toennesen, First detection of charged dust particles in the Earth's mesosphere, J. Geophys. Res., 101, 10,839-10,847, 1996.

Havnes, O., A. Brattli, T. Aslaksen, W. Singer, R. Latteck, T. Blix, E. Thrane, and J. Troeim, First common volume observations of layered plasma structures and polar mesospheric summer echoes by rocket and radar, Geophys. Res. Lett., 28, 1419-1422, 2001.

Horanyi, M., S. Robertson, B. Smiley, J. Gumbel, G. Witt, and B. Walch, Rocketborne mesospheric measurement of heavy ( $\mathrm{m} / \mathrm{gg} 10 \mathrm{amu}$ ) charge carriers, Geophys. Res. Lett. 27, 3821-3825, 2000.

Jensen, E. and G.E. Thomas, Growth-sedimentation model of polar mesospheric clouds: comparison with SME measurements, J. Geophys. Res., 93, 2461-2473, 1988.

Lebedinets, V.N., Dust in the upper atmosphere and in space. Meteors, Gidrometeoizdat, Leningrad, 1980, 271 pp., (in Russian).

Maynard, N.C., C.L. Croskey, J.D. Mitchel, and L.C. Hale, Measurements of volt/meter vertical electric fields in the middle atmosphere, Geophys. Res. Lett., 8, 923-926, 1981.

Mitchel, J.D., Electrical properties of the middle atmosphere, Adv. Space Res., 10, 219-228, 1990.

Tyutin, A.A., Mesospheric maximum of the electric field strength, Cosmic Res., Eng. Trans., 14, 132-133, 1976.

Zadorozhny, A.M., Tyutin, A.A., G. Witt, N. Wilhelm, U. W., Y.J. Cho, and W.E. Swartz, Electric field measurements in the vicinity of noctilucent clouds and PMSE, Geoph. Res. Lett., 20, 2299-2302, 1993.

Zadorozhny, A.M., Tyutin, A.A., O.A. Bragin, and Y.N. Kikhtenko, Recent measurements of middle atmospheric electric fields and related parameters, $J$. Atmos. Terr. Phys., 56, 321-335, 1994.

Zadorozhny, A.M., On the role of charged dust in mesospheric electric fields Geoph. Res. Lett., 27, 493-496, 2000. 